\title{
Prophylaxis of migraine: general principles and patient acceptance
}

\author{
Domenico D'Amico' \\ Stewart J Tepper ${ }^{2}$ \\ 'Headache Center, Department \\ of Neurological Sciences, C Besta \\ Neurological Institute, Milan, Italy; \\ ${ }^{2}$ Center for Headache and Pain, \\ Neurological Institute, Cleveland \\ Clinic Foundation, Cleveland, \\ Ohio, USA
}

\begin{abstract}
Migraine is a chronic neurological condition with episodic exacerbations. Migraine is highly prevalent, and associated with significant pain, disability, and diminished quality of life. Migraine management is an important health care issue. Migraine management includes avoidance of trigger factors, lifestyle modifications, non-pharmacological therapies, and medications. Pharmacological treatment is traditionally divided into acute or symptomatic treatment, and preventive treatment or prophylaxis. Many migraine patients can be treated using only acute treatment. Patients with severe and/or frequent migraines require long-term preventive therapy. Prophylaxis requires daily administration of anti-migraine compounds with potential adverse events or contraindications, and may also interfere with other concurrent conditions and treatments. These problems may induce patients to reject the idea of a preventive treatment, leading to poor patient adherence. This paper reviews the main factors influencing patient acceptance of anti-migraine prophylaxis, providing practical suggestions to enhance patient willingness to accept pharmacological anti-migraine preventive therapy. We also provide information about the main clinical characteristics of migraine, and their negative consequences. The circumstances warranting prophylaxis in migraine patients as well as the main characteristics of the compounds currently used in migraine prophylaxis will also be briefly discussed, focusing on those aspects which can enhance patient acceptance and adherence.
\end{abstract}

Keywords: migraine, prophylaxis, preventive therapy, acceptance, adherence

\section{Introduction}

Migraine can be regarded as a chronic neurological condition with episodic exacerbations. Migraine is highly prevalent, and associated with significant pain, disability, and diminished quality of life. Thus, migraine management is an important health care issue.

Different elements need to be considered in migraine management. They include: avoidance of trigger factors, lifestyle modifications, non-pharmacological therapies, and medications. Pharmacological treatment is traditionally divided into acute or symptomatic treatment, and preventive treatment or prophylaxis. Many migraine patients can be treated using only acute treatment, that is, prescribing medications that are used only during headache attacks to abort an ongoing attack or to stop its progression to severe pain and associated symptoms. Patients with severe and/or frequent migraines require long-term preventive therapy.

Prophylaxis requires daily administration of anti-migraine compounds, whether or not a migraine attack is occurring. All the drugs used for migraine prevention have potential and often relevant adverse events or contraindications, and may also interfere with other concurrent conditions and treatments. These problems may induce patients to reject the idea of a preventive treatment, or may lead to poor patient adherence.

This paper reviews the main factors influencing patient acceptance of anti-migraine prophylaxis, providing some practical suggestions to enhance patient's willingness 
to accept pharmacological anti-migraine preventive therapy. We also provide information about the main clinical characteristics of migraine and on the negative consequences of this condition. The circumstances warranting prophylaxis in migraine patients as well as the main characteristics of the compounds currently used in migraine prophylaxis will also be briefly discussed, focusing on those aspects which can enhance patient acceptance and adherence

\section{Migraine overview}

\section{Clinical presentation of migraine}

Migraine is an idiopathic headache disorder with a high prevalence globally (Stewart et al 1994; Launer et al 1999; Roncolato et al 2000; Lipton et al 2001; Henry et al 2002; Wang 2003; Steiner 2003; Lipton et al 2007). About 6\% to $8 \%$ of men and $12 \%$ to $14 \%$ of women meet criteria for migraine. The disorder is characterized by episodes of moderate to severe head pain, which is often unilateral and pulsating, and typically aggravated by routine physical activities. The duration of untreated migraine attacks is rather long, from 4 hours to 3 days (median duration 18 hours). Pain is accompanied by autonomic symptoms, the most common being nausea, vomiting, photophobia, and phonophobia (Headache Classification Subcommittee of the International Headache Society 2004). Other symptoms, such as osmophobia, pallor, fatigue, difficult in concentration, blurred vision, or diarrhea, can be present.

In many patients the headache phase is preceded by premonitory symptoms (or prodromes) which can last from a few hours to 24 hours, and are characterized by fatigue, yawning, fluid retention, mood changes, sensory hypersensitivity, food cravings, or increased thirst (Blau 1984). Similar psychological, general and autonomic symptoms may also characterize the resolution phase of an attack (postdrome) (Blau 1980).

For up to $25 \%$ of migraineurs, the headache phase can be preceded by transient focal neurological symptoms, generally lasting from 4 minutes to 1 hour, defined as migraine aura. The most common aura symptoms are visual disturbances (scintillating scotomata, hemianopia, blind spots), sensory disturbances (unilateral paresthesias affecting the face and limbs, numbness), or speech/language disturbances (dysarthria, aphasia). Uncommon aura phenomena include weakness, ataxia, vertigo, or loss of consciousness (Headache Classification Subcommittee of the International Headache Society 2004). The median migraine attack frequency is one per month, although about $30 \%$ of sufferers in the general population report three or more attacks per month (Lipton et al 2007).
Some attacks are unpredictable, but in most patients several factors which are able to trigger migraine attacks are evident (Silberstein 1990). Migraine triggers include dietary factors (such as hunger, alcohol, and chocolate), hormonal changes (menstruation), environmental factors (such as bright lights, odors, and altitude), physical activities (sex, sports), and psychological and chronobiological changes (stress, sudden anxiety or joy, too much or too little sleep, schedule changes).

There is a wide variability in the features and in the frequency of attacks, as well as in the burden of disease on the individual (Stewart et al 1994). This variation depends on constitutional and environmental factors and on coexisting conditions, and explains why therapeutic needs differ among patients and often in the same patient at different periods of his or her life, justifying tailored migraine management.

\section{Influence of migraine on functioning and on quality of life}

The significant reported clinical characteristics of migraine, and the fact that its prevalence and incidence peak in middle life when an individual is likely to be more actively engaged in work, social and family duties, explains why migraine can cause a significant negative impact on patient lives (Stewart et al 1994; Roncolato et al 2000; Lipton et al 2001; Henry et al 2002; Lipton et al 2007). Numerous studies have demonstrated that migraine is very disabling, often causing severe functional impairment during attacks.

The majority of people with migraine in the general population sampled in the population-based second American Migraine Study (AMS II) (Lipton et al 2001) experienced some form of functional impairment. Fifty-three percent reported that their severe headaches led to substantial impairment of daily activities and bed rest in many cases, while 1 of every 3 migraineurs missed at least 1 day of work or school in the 3 months prior to the survey.

For 264 consecutive migraine patients at an Italian Headache Center (D'Amico et al 2004), all daily activities were reportedly impaired. The mean values obtained in this survey indicated that over the 3-month period preceding the survey, total or partial impairment due to migraine was evident in all daily duties. The mean values obtained in this survey indicated that ability to perform housework and personal and social activities were affected by migraine on 14.5 days/3 months (household work missed on 4.3 days; household significantly impaired on 4.6 days; social, leisure and family duties skipped on 5 days). During the same period of time, total or partial disability affected patients' 
occupational activities on a mean of 8.8 days $/ 3$ months, with 2.2 days of absenteeism (ie, days with absence from work or from school because of attacks), and 6.6 days of presenteeism (ie, patients experiencing a significant reduction in work or school productivity because of migraine attacks while they were at work or at school) (D’Amico et al 2004).

A telephone survey with 6-month recall conducted in Israel and eight European countries on young women with migraine, who were employed or full-time students, showed that during the prior 6 months, $46 \%$ of participants missed at least 1 day of work or school, and $74 \%$ were prevented from functioning fully at work or school because of migraine. Mean work/school absenteeism due to migraine was 1.9 days over 6 months (range, 0.8-2.8 days). More than $60 \%$ of young migraineurs reported one or more occurrences of being unable to spend time with family and friends or unable to enjoy recreational activities (Dueland et al 2004).

The pervasive disruption of migraine on all daily duties was confirmed in French surveys on the general population (Lucas et al 2005; Lucas et al 2006). In population-based surveys from the USA and UK (Lipton et al 2003), 85\% of migraineurs living with a partner reported substantial reduction during migraine in their ability to do household work. Forty-five percent were forced to miss social, family, and leisure activities, and 32\% avoided making plans for fear of cancellation due to possible occurrence of a migraine attack. In the same studies, $50 \%$ of the interviewed migraineurs believed that they were more likely to argue with their partners and children, and many of them reported a negative influence of the disorder on time spent with their children and partners, reporting elements of disharmony and conflict in their families because of migraine (such as deciding not to have children or delaying having children). Negative consequences on work performances, time spent in family activities, difficulty in communication and, as noted, tendency to argue with partners were also reported by the household partners of individuals with migraine.

Migraine can cause significant life style changes. Besides family engagements, education, employment choices and achievements may also be curtailed both by unpredictable attacks and by the tendency of migraineurs to avoid certain situations which involve the risk of triggering a migraine attack.

In addition, these factors lead to a negative impact of migraine in between attacks. Numerous studies have confirmed that migraineurs experience a poor quality of life and diminished feelings of well-being even during the pain-free intervals. Studies performed in different countries, either on individuals from the general population or on patients from headache clinics, reveal that migraine is associated with significantly lower scores on various health-related quality-of-life rating scales, regardless of age, gender, or socioeconomic status (Osterhaus et al 1994; Monzón and Lainez 1998; Terwindt et al 2000; D’Amico et al 2004; Lucas et al 2006). Patients with migraine report disturbances of sleep and contentment, and experience greater emotional distress compared to non-migraine subjects (Dahlof and Dimenas 1995). Health-related quality of life in migraineurs is worse than that observed in people without migraine, and it appears to be similar in physical functions, and even worse in social and emotional domains, than in people with other chronic disorders, such as myocardial infarction, diabetes, hypertension, and asthma (Osterhaus et al 1994; Terwindt et al 2000).

\section{Preventive therapy in migraine patients Indications and goals of prophylaxis}

While acute treatment is generally indicated in all migraineurs, prophylaxis is indicated only in selected cases. The universally accepted goal of prophylaxis is a reduction in migraine frequency. It is important to note that in recent years it has become clear that this aspect cannot be the only indicator of treatment success and patient satisfaction (Silberstein for the US Headache Consortium 2000; Dowson et al 2004; Sheftell et al 2004; Members of the task force EFNS 2006; D’Amico and Lanteri-Minet 2006; Diamond et al 2006).

International evidence-based guidelines from the US Headache Consortium (Silberstein SD for the US Headache Consortium 2000) and the European Federation of Neurological Societies (EFNS) (Members of the task force EFNS 2006) have focused on the circumstances warranting preventive treatment in migraineurs. Besides a high mean monthly number of attacks, a decision to start a preventive treatment in an individual migraineur must be guided by the effectiveness of acute therapy in that individual, the risk of medication overuse, by his/her preference to start a daily treatment, and, above all, by the impact of the condition on a patient's daily life (Tables 1, 2).

Appropriate prophylaxis can reduce the global burden of migraine. D'Amico et al (2006) showed that both ability to perform daily activities (assessed by MIDAS), and healthrelated quality of life (investigated by SF-36) can improve significantly in migraine patients receiving different currently used preventive drugs for a period of three months. Data from controlled trials with topiramate in migraine prophylaxis 
Table I US headache consortium guidelines: circumstances warranting preventive medications in migraineurs (Silberstein 2000)

I. Recurring migraine that significantly interferes with the patient's daily routine despite acute treatment (eg, two or more attacks a month that produce disability that lasts at least 3 days or headache attacks that are infrequent but produce profound disability);

2. Failure, contraindication to, or troublesome side-effects from acute medications;

3. Overuse of acute medications;

4. Special circumstances, such as hemiplegic migraine or attacks with a risk of permanent neurological injury;

5. Very frequent headaches (more than two a week), or a pattern of increasing attacks over time, with the risk of developing medication overuse headache or rebound with acute attack medicines;

6. Patient preference, ie, the desire to have as few acute attacks as possible.

suggest that quality of life was better in treated patients, when measured with a generic questionnaire (SF-36) and with a migraine-specific tool (MSQ) (Brandes et al 2006; Dahlof et al 2007), and also that disability might be reduced when patients received this drug for periods of 6 to 14 months (Diener et al 2007).

\section{General principles of prophylaxis}

Typically, preventive drugs must be used for periods of months. International guidelines suggest a minimum trial of 2 to 3 months of daily administration, which in fact is the shortest time period needed to assess clinically relevant effects on the condition (Silberstein for the US Headache Consortium 2000; Members of the task force EFNS 2006). Although there is not a general agreement on the ideal duration of each prophylaxis period, recently published data suggest greater opportunity with longer treatment periods. The efficacy of topiramate in migraine prevention was maintained when this compound was administered continuously for up to 14 months (Diener et al 2007). In another study, the efficacy of topiramate was further increased when prophylaxis was continued for rather long periods (Rapoport et al 2006). When a group of migraine patients were treated for 8 months in an open-label extension

Table 2 EFNS Task Force Guidelines (Members of the task force EFNS 2006): factors that indicate the opportunity of consider and discuss anti-migraine prophylaxis with the patient

- The quality of life, business duties, or school attendance are severely impaired

- Frequency of attacks per month is two or higher

- Migraine attacks do not respond to acute drug treatment

- Frequent, very long, or uncomfortable auras occur phase after two large double-blind, placebo-controlled trials of 26 weeks duration, the mean number of headache attacks decreased from $3.4+/-2.6$ per month in patients treated with topiramate (at the end of the double-blind treatment periods) to $2.2 \pm 2.4$ per month after completion of the open-label phase with the active drug (Rapoport et al 2006).

\section{Available compounds for anti-migraine prophylaxis}

In 2000, the US Headache Consortium published technical reports and guidelines on preventive medications for migraine. (Silberstein for the US Headache Consortium 2000: Ramadan et al 2000). These reports evaluated preventive agents by strength of evidence, scientific effect measures, and clinical impression of effect. These evaluations were collected into groups as seen in Table 3.

Since this publication, evidence for topiramate in the first group, and several other medications that would properly be placed in Group 2 have been published. These will be summarized below.

\section{Mechanisms of action}

Migraine is a generally inherited condition of neuronal hyperexcitability with a central generator, peripheral pain mechanisms, and central integration. Debate continues as to whether the generator for migraine is due to cortical spreading depression (CSD), that is, is cortical in location and pathophysiology, or whether CSD occurs just in those with aura, while those without aura have the onset of migraine in a central brainstem periaquductal gray/dorsal raphe midbrain generator.

CSD begins with a slow activation of neurons and glia and associated hyperemia. This activation is in turn followed by a wave of neuronal depression, accompanied by oligemia. CSD activates brain matrix metalloproteinases (MMP), such as MMP-9, opening the blood brain barrier, which may be necessary for preventive medications accessing central brain compartments (Gursoy-Ozdemir et al 2004).

Ayata et al published a seminal paper in 2006, in which topiramate, valproate, propranolol, amitriptyline, methysergide, saline, or D-propranolol (clinically ineffective for migraine and serving as a second placebo) were administered to rats either acutely or chronically over weeks and months. The impact of treatment was determined on the frequency of evoked CSDs after topical potassium application or on the incremental cathodal stimulation threshold to evoke CSD. Chronic daily administration of migraine prophylactic drugs dose-dependently suppressed CSD frequency by $40 \%$ to $80 \%$ 
Table 3 Preventive medications as listed by the US Headache Consortium (Ramadan et al 2000)

\begin{tabular}{|c|c|c|c|c|}
\hline $\begin{array}{l}\text { Group I: } \\
\text { Medium to high efficacy, } \\
\text { good strength of evidence, } \\
\text { and a range of severity } \\
\text { (mild to moderate) and } \\
\text { frequency (infrequent to } \\
\text { frequent) of side effects }\end{array}$ & $\begin{array}{l}\text { Group 2: } \\
\text { Lower efficacy than } \\
\text { those listed in first } \\
\text { column, or limited } \\
\text { strength of evidence, } \\
\text { and mild to moderate } \\
\text { side effects }\end{array}$ & $\begin{array}{l}\text { Group 3: } \\
\text { Clinically efficacious } \\
\text { based on consensus and } \\
\text { clinical experience, but } \\
\text { no scientific evidence } \\
\text { of efficacy }\end{array}$ & $\begin{array}{l}\text { Group 4: } \\
\text { Medium to high } \\
\text { efficacy, good strength } \\
\text { of evidence, but with } \\
\text { side effect concerns }\end{array}$ & $\begin{array}{l}\text { Group 5: } \\
\text { Evidence indicating } \\
\text { no efficacy over } \\
\text { placebo }\end{array}$ \\
\hline Amitriptyline & Aspirin $^{\#}$ & a. mild-to-moderate & Methysergide & Acebutolol \\
\hline Divalproex sodium & Atenolol & side effects & Flunarizine* & Alprenolol* \\
\hline Lisuride* & Cyclandelate* & Cyproheptadine & Pizotifen* & Carbamazepine \\
\hline Propranolol & Fenoprofen & Bupropion & TR-DHE* & Clomipramine, \\
\hline \multirow[t]{18}{*}{ Timolol } & Feverfew & Diltiazem & & Clonazepam \\
\hline & Flurbiprofen & Doxepin & & Clonidine DEK* \\
\hline & Fluoxetine (racemic) & Fluvoxamine & & Femoxetine* \\
\hline & Gabapentin & Ibuprofen & & Flumedroxone* \\
\hline & Guanfacine & Imipramine & & Indomethacin \\
\hline & Indobufen* & Mirtazepine & & Iprazochrome* \\
\hline & Ketoprofen & Nortriptyline & & Lamotrigine \\
\hline & Lornoxicam* & Paroxetine & & Mianserin* \\
\hline & Magnesium & Protriptyline & & Nabumetone \\
\hline & Mefenamic acid & Sertraline & & Nicardipine \\
\hline & Metoprolol & Tiagabine & & Nifedipine \\
\hline & Nadolol & Topiramate & & Oxprenolol* \\
\hline & Naproxen sodium & Trazodone & & Oxitriptan* \\
\hline & Nimodipine & Venlafaxine & & Pindolol \\
\hline & Tolfenamic acid* & & & Tropisetron* \\
\hline & Verapamil & b. (side effect concerns) & & Vigabatrin* \\
\hline & Vitamin B2 & $\begin{array}{l}\text { Methylergonovine } \\
\text { (methylergometrine) }\end{array}$ & & \\
\hline & & Phenelzine & & \\
\hline
\end{tabular}

"Does not include combination products.

*Currently not available in US.

and increased the cathodal stimulation threshold, whereas acute treatment was ineffective. Longer treatment durations produced stronger CSD suppression. Chronic D-propranolol treatment did not differ from saline control. The study suggested that CSD may be the common therapeutic target for widely prescribed migraine prophylactic drugs (Ayata et al 2006).

Migraine pain itself is caused by neurogenic inflammation and vasodilation in the meninges, sensitizing nociceptive afferents. The central generator somehow activates these peripheral pain mechanisms, and migraine preventive agents likely share common inhibitory effects which decrease central neuronal hyperexcitability, making the initiation of these inflammatory and vascular pain events less frequent and less severe.

\section{Classes of migraine medications Tricyclic antidepressants (TCAs)}

Amitriptyline, a tertiary amine TCA, as noted in Table 4, has excellent quality of evidence for effect, $3+$ scientific and clinical impression of effect, and is in Group 1 of the US Headache Consortium Guidelines (Ramadan et al 2000). Amitriptyline is registered as a migraine preventive medication in the UK, in Italy, and other countries, but not in the US.

Nortriptyline, protriptyline (tertiary amines), doxepin (secondary amine), and imipramine have clinical impression for efficacy but no or limited randomized controlled trials (RCTs) (Ramadan et al 2000).

Adverse events associated with the use of TCAs are best described as "the four horsemen of the apocalypse": dry mouth, constipation, sedation, and weight gain, the latter from antihistaminic effects. Dry mouth, constipation, tachycardia, blurry vision, and urinary retention are all anticholinergic side effects, maximal in the elderly. TCAs can lower the seizure threshold and can cause cardiac arrhythmias and orthostatic hypotension. Overdose is frequently lethal. TCAs can cause the syndrome of inappropriate ADH secretion (SIADH), and precipitate mania in bipolar patients. 
Table 4 Tricyclic antidepressants (TCAs) in migraine prevention

\begin{tabular}{llll}
\hline TCA & $\begin{array}{l}\text { Recommended } \\
\text { dose/day }\end{array}$ & $\begin{array}{l}\text { US Headache } \\
\text { Consortium } \\
\text { Quality of } \\
\text { evidence (*) }\end{array}$ & $\begin{array}{l}\text { US Headache } \\
\text { Consortium } \\
\text { Clinical } \\
\text { effectiveness (*) }\end{array}$ \\
\hline Amitriptyline & $30-150 \mathrm{mg}$ & $\mathrm{A}$ & $3+$ \\
Nortriptyline & $25-100 \mathrm{mg}$ & $\mathrm{C}$ & $3+$ \\
$\begin{array}{l}\text { Doxepin, } \\
\text { Imipramine }\end{array}$ & $30-150 \mathrm{mg}$ & $\mathrm{C}$ & + \\
Protriptyline & $10-40 \mathrm{mg}$ & $\mathrm{C}$ & $2+$ \\
\hline
\end{tabular}

(*) Ratings from Ramadan et al 2000.

Maximizing treatment of comorbid illnesses would suggest selection of TCAs for use in patients with comorbid depression, sleep disturbance, or neck pain.

\section{Beta blockers}

Propranolol, a non-selective beta-blocker, has top level effectiveness and scientific evidence (Ramadan et al 2000), and is registered for migraine prevention in the US, UK, Italy, and other countries. Timolol, also non-selective, is also approved for migraine prevention in the US, and has almost as good evidence for efficacy as propranolol (Ramadan et al 2000).

Nadolol, a non-selective beta-blocker, and atenolol and metoprolol, beta-1 selective beta-blockers are listed in Group 2 by the US Headache Consortium (Ramadan et al 2000). The responder rate for beta blockers, those with at least a $50 \%$ reduction of migraine frequency, is $43 \%$ to $80 \%$.

Quality of evidence and recommended daily doses are reported in Table 5. Beta blockers are relatively contraindicated in patients with hypotension, congestive heart failure, asthma, Raynauds, depression, and diabetes mellitus.

Beta blockers should be considered in patients with comorbid hypertension or anxiety but not depression.

Table 5 Beta-blockers in migraine prevention

\begin{tabular}{llll}
\hline Beta blocker & $\begin{array}{l}\text { Recommended } \\
\text { dose/day }\end{array}$ & $\begin{array}{l}\text { US Headache } \\
\text { Consortium } \\
\text { Quality of } \\
\text { evidence (*) }\end{array}$ & $\begin{array}{l}\text { US Headache } \\
\text { Consortium } \\
\text { Clinical } \\
\text { Effectiveness (*) }\end{array}$ \\
\hline Propranolol & $80-240 \mathrm{mg}$ & $\mathrm{A}$ & $3+$ \\
Timolol & $20-30 \mathrm{mg}$ & $\mathrm{A}$ & $2+$ \\
Atenolol & $100 \mathrm{mg}$ & $\mathrm{B}$ & $2+$ \\
Nadolol & $80-240 \mathrm{mg}$ & $\mathrm{B}$ & $3+$ \\
Metoprolol & $200 \mathrm{mg}$ & $\mathrm{B}$ & $3+$ \\
\hline
\end{tabular}

(*) Ratings from Ramadan et al 2000.

\section{Calcium channel blockers}

Flunarizine is the most widely used calcium channel blocker world wide. Although it is not available in the US, it is one of the most prescribed preventive drugs in Italy and in other European countries, as well as in Latin America. It is very effective clinically, with adequate, Grade B scientific evidence for $10 \mathrm{mg}$ /day but the US Headache Consortium notes "side effect concerns, due to the risk of sedation, weight gain, depression, and extrapyramdal signs" (Ramadan et al 2000). These effects can be reduced by using the $5 \mathrm{mg}$ /day dose.

Verapamil at $240 \mathrm{mg} /$ day and diltiazem have similar level of scientific evidence to flunarazine, with much less evidence of clinical effectiveness. The other calcium channel blockers have even less or no RCTs on which to rely (Ramadan et al 2000).

Some of the hemiplegic migraine subtypes and some patients with typical aura have been demonstrated to have calcium channelopathies, so that calcium channel blockers are more often selected in prevention for aura patients.

Cardiac effects from some calcium channel blockers (namely, from verapamil) are of concern, and may merit monitoring of the QTc intervals on cardiograms. Also described are atrioventicular block, bradycardia, and arrhythmias. Constipation is common with verapamil, and hypotension, and peripheral edema may occur as well (Silberstein and Goadsby 2002).

\section{Anti-epilepsy drugs (AEDs) Valproate}

Valproate has excellent scientific evidence, clinical effectiveness, and is registered for migraine prevention in the US and other countries (not in Italy) for doses from 500 to $1500 \mathrm{mg}$ /day. The levels of scientific evidence and clinical evidence are reported in Table 6.

The limitations of valproate are its potential for serious adverse events. Teratogenicity, with neural tube defects occurs at a rate of $1 \%$ to $2 \%$. In addition, polycystic ovarian syndrome occurred in $10.5 \%$ of female menstruating patients within one year of initiation of valproate treatment (Joffe et al 2006). The authors believe that valproate should not be used as a first line treatment in migraine prevention for young, menstruating women.

Other common adverse events in order of occurrence are nausea, asthenia, and dyspepsia. Hepatotoxicity is common in children under 2 years old, but increases in likelihood in adults when valproate is co-administered with medications that induce the cytochrome p450 system such as barbiturates and benzodiazepines. Therefore, valproate is best 
Table 6 AEDs in migraine prevention

\begin{tabular}{llll}
\hline AED & $\begin{array}{l}\text { Recommended } \\
\text { dose/day }\end{array}$ & $\begin{array}{l}\text { Level of scientific } \\
\text { evidence }\end{array}$ & $\begin{array}{l}\text { Level of clinical } \\
\text { effectiveness }\end{array}$ \\
\hline Valproate & $500-1500 \mathrm{mg}$ & $\mathrm{A}$ & $3+$ \\
Gabapentin & $900-2400 \mathrm{mg}$ & $\mathrm{B}$ & $2+$ \\
Topiramate & $100 \mathrm{mg}$ & $\mathrm{A}$ & $3+$ \\
\hline
\end{tabular}

administered in prophylaxis as monotherapy. Other adverse events of concern include alopecia, tremor, and weight gain. Since valproate can rarely cause thrombocytopenia, other bone marrow dysfunction, and pancreatitis, in addition to the hepatic problems, blood monitoring is recommended.

\section{Gabapentin}

Gabapentin has adequate scientific evidence for effectiveness at $2400 \mathrm{mg}$ /day based on one RCT. It has a propensity for causing drowsiness and dizziness, but no drug-drug interactions, and is excreted unchanged in the urine (Mathew et al 2001). The levels of scientific evidence and clinical evidence, and the recommended daily dose, are reported in Table 6.

\section{Topiramate}

Topiramate has excellent scientific evidence and clinical evidence for effectiveness, and is registered for migraine prevention in the US and most countries with $100 \mathrm{mg}$ the optimal dose (Brandes et al 2004; Silberstein et al 2004). The levels of scientific evidence and clinical evidence are reported in Table 6.

CNS side effects are the most worrisome adverse events with topiramate use and include language problems, memory difficulty, and decreased concentration. Other serious adverse events include rare narrow angle-closure glaucoma early in treatment, hyperchloremic acidosis in $>10 \%$ of patients, nephrolithiasis at a rate of $1 \%$ due to carbonic anhydrase inhibition, and rare oligohydrosis, which can result in fatal hyperthermia, more frequently in younger age groups at higher doses (Ziad et al 2005).

Around half of patients treated with topiramate will experience paresthesias. Weight loss, usually a welcome side effect, occurred in $9 \%$ of patients at $100 \mathrm{mg}$, the optimal dose, and the mean weight loss in those losing weight over a year was $3 \%$. Other side effects can include fatigue and diarrhea.

\section{Anti-serotonin drugs}

Two commonly used medications that block serotonin $(5-\mathrm{HT})_{2}$ receptors are cyproheptadine (available in the US and some countries) and pizotifen (not available in the US, but available in most European countries, including Italy).
Both are described by the US Headache Consortium as having "medium to high efficacy, good strength of evidence, but with side effect concerns", namely weight gain and drowsiness (Ramadan et al 2000). The dose for cyproheptadine is 4 to $12 \mathrm{mg} /$ day, for pizotifen, 1.5 to $6 \mathrm{mg}$ /day.

Two other 5- $\mathrm{HT}_{2}$ antagonists are ergots, methysergide and methylergonovine or methylergometrine, its active metabolite. Evidence for the former is excellent, for the latter anecdotal.

These long acting ergots are less commonly used in migraine prevention due to the fact that triptans cannot be used as acute treatment in patients on ergots preventively and due to the risk of idiosyncratic fibrosis in heart (including valvular fibrosis and resultant regurgitation and heart failure), GI tract, and lung, which can occur in $1 / 1500$ to 1/5000 patients after 6 months of steady use of methysergide. The risk for fibrotic complications has been linked to $5-\mathrm{HT}_{2 \mathrm{~B}}$ agonist effects, despite the overall hypothesis that these medications work as 5- $\mathrm{HT}_{2}$ antagonists (Roth et al 2007). The authors use drug holidays every 6 months based on the hope rather than any evidence that this will prevent fibrotic complications for both medications. Those patients on these medications chronically should be monitored with CT of abdomen and lungs, and echocardiography. The fibrotic risk is probably why methysergide is not longer available in the US and Italy.

Other side effects can include drowsiness, leg aches presumably from ergotism, and GI adverse events of nausea, pain, and diarrhea.

Doses are up to $6 \mathrm{mg} /$ day for methysergide and up to $0.6 \mathrm{mg} /$ day for methylergonovine/methylergometrine.

\section{Herbs, minerals, vitamins, and supplements}

Petasites (butterbur root), magnesium, vitamin B2 (riboflavin), and coenzyme Q10 all have adequate second level randomized controlled scientific and clinical evidence for efficacy. Doses are $150 \mathrm{mg} /$ day of petasites, 400 to $600 \mathrm{mg} /$ day of chelated magnesium, 25 to $400 \mathrm{mg} /$ day of vitamin B2, and $300 \mathrm{mg} / \mathrm{day}$ of coenzyme Q10. Adverse events include eructation and GI upset for petasites, diarrhea for magnesium, bright yellow urine and rash for vitamin B2, and rash with coenzyme Q10 (Lipton et al 2004; Sandor et al 2005; Tepper et al 2006).

\section{Other preventive agents}

There is second level evidence of effectiveness for aspirin, and certain NSAIDs such as naproxen and ketoprofen. The same level of evidence (eg, single RCTs, or multiple 
less definitive RCTs) exists for the angiotensin converting enzyme inhibitor lisinopril at $20 \mathrm{mg} /$ day, the angiotensin receptor blocker candasartan at $16 \mathrm{mg} /$ day, and for the serotonin norepinephrine inhibitor venlafaxine at $150 \mathrm{mg} /$ day.

\section{Starting, selecting and using preventive medication}

The decision to use daily preventive is based on a weighing of frequency of attacks and acute medication use on the one hand, and disability or impact on the other. As number of headache days increases and number of days in which a patient used acute medication increases, the risk of transformation into daily headache also increases dramatically. At around 9 days of acute medication use per month, or 10 days of headache per month, the risk for chronification of headache becomes so great that daily preventive treatment becomes essential, to drive the number of headache days down, allowing for judicious use of acute treatments and preventing medication overuse headache (Scher et al 2003; Katsarava et al 2004; Bigal et al 2006).

Daily prevention can be initiated at far lower frequencies of headache if disability is high or response to acute medication poor. This can be the case when triptans and ergots are contraindicated due to vascular disease, a situation calling for earlier intervention with daily preventive medication.

Special circumstances in which the risk for adverse outcomes from migraine appear significant, such as prolonged aura, or when each attack carries profound impact, such as in hemplegic and basilar-type migraine, also necessitate daily preventive medication.

Silberstein and Goadsby suggest the following approaches to successful preventive treatment:

1. Start the drug at a low dose.

2. Give each treatment an adequate trial.

3. Avoid interfering, overused, and contraindicated drugs.

4. Re-evaluate therapy.

5. Be sure that women of childbearing potential are aware of any potential risks.

6. Involve patients in their care to maximize compliance.

7. Consider comorbidity and choose medications to treat several coexisting disorders where possible.

8. Choose a drug based on its proven efficacy, the patient's preferences and headache profile, the drug's side effects, and the presence or absence of coexisting or comorbid disease (Silberstein and Goadsby 2002).

Our own recommendations are:

1. It is always best to try to select preventive medication calculated to treat multiple comorbid medical or psychiatric illnesses at the same time. Examples of this include migraine patients with sleep disturbance, depression, and neck pain, treated optimally with amitriptyline; or hypertension and anxiety, treated optimally with a beta blocker (Dowson et al 2003; Tepper et al 2004; Silberstein et al 2007).

2. Do not forget that patients administered daily preventive medication must also be provided with acute medication, preferably migraine-specific. The severity of their migraine has led to the need for prophylaxis, they will have breakthrough migraines, and they must be given adequate as-needed treatment at the same time, with limits on usage to avoid medication overuse headache.

3. Patients who have chronic daily headache or medication overuse headache, that is, headaches $>15$ days per month, will most likely respond neither to daily preventive medications nor triptans. Before initiation of preventive therapy, detoxification is necessary; a failure to detoxify patients in medication overuse headache before starting preventive therapy is one of the most common reasons prophylaxis fails, along with starting at too high a dose, and failure to keep a patient on prevention for a long enough time.

\section{Factors that can influence migraine patient acceptance of prophylaxis Daily administration of preventive drugs}

Patients might reject the idea of taking a pill each and every day, independently from the occurrence of their attacks. One reason for this is because they view migraine as an episodic disorder.

Physicians should communicate that, although characterized by episodic attacks of head pain and associated symptoms, migraine is in fact a chronic condition. Care providers should provide some explanations about the biology of migraine and the role of prophylaxis. They can make patients aware that migraine is a physiologic disorder with a constitutional predisposition and a particular susceptibility to develop the individual headache attacks when migraineurs are exposed to several biological and environmental triggering factors, and that consequently the condition may be partially corrected by the use of preventive medications which can modulate this "neurochemical instability."

\section{Long duration of preventive therapy}

Patients might not accept taking preventive drugs for periods of at least several months. Explanations on the nature of migraine as well as on the goals and the mechanisms of action 
of preventive compounds are required. This information should help patients to recognize that prophylaxis is by definition a long-term treatment, as its rationale is to gradually exert a positive action on the complex mechanisms causing migraine, and that the effect of prevention requires a regular and continuous administration for relatively long therapeutic cycles.

\section{Definition and assessment of clinical outcomes}

Some patients may expect that migraine prevention will fully eradicate their headaches. Others might think that their condition cannot be neither cured nor relieved by pharmacological interventions, and that they must "keep on suffering", with no hope of improvement.

Physicians must inform patients that both of these concepts are not correct. It is extremely important that care providers should provide realistic expectations for treatment outcomes, based on scientific evidence and on their own clinical experience. This means explaining that reduction in frequency is the primary goal, with spill over benefits on intensity and duration, as well as disability, but that acute attacks will still occur even with successful prophylaxis.

Physicians therefore should deliberately and clearly explain that scientific evidence shows that the drugs currently used in migraine prophylaxis are effective when compared to placebo, and that subjects on preventive compounds are likely to see a reduction in their headache episodes, as well as in their intensity and duration. It is important to share with them the main results of recent studies, indicating that prophylaxis is also able to restore their functioning in between attacks, by decreasing the negative effects of the condition on ability to perform daily activities and on quality of life.

As noted, patients must be carefully informed that complete disappearance of attacks cannot be expected after prophylaxis, and that analysis of data from the published literature indicates that about $45 \%$ of migraineurs receiving available preventive drugs will experience a reduction in the mean monthly frequency of their attacks of $50 \%$ or more, and around $20 \%$ of them will see a frequency reduction of $75 \%$ or more (Klapper 1997; Mathew et al 2001; Brandes et al 2004; Diener et al 2004; Silberstein et al 2004).

Patients should be encouraged to keep a headache diary in order to record the occurrence of attacks during the treatment periods, inviting them to provide information also about the severity of pain and the degree of functional disability during their migraines. This will favor communication with their physician about the effectiveness of the ongoing treatment, and eventually indicate the need for adjusting daily doses or for changing the current preventive drug.

This line of communication could also be enhanced by the use of specific questionnaires to assess the impact of migraine on functioning and on the patient's sense of well being. As noted above, clinicians should focus the attention of their patients on the fact that prophylaxis can eventually be useful in reducing migraine-related burden, and that reduction in disability and improvement in quality of life have been found in some clinical studies, and that these outcomes can be also be incorporated into clinical practice. In fact, clinicians can use specific questionnaires to assess the efficacy of prophylaxis in a given patient. Among numerous tools developed and tested in recent years, the Migraine Disability Assessment score (MIDAS) (Stewart et al 1999), and the Headache Impact test (HIT6) (Kosinski et al 2003) are suitable for clinical office use, as they are both simple, brief, self-administered, easy-to-score questionnaires.

MIDAS has five questions assessing headache-related disability over the preceding 3 months, and can be summarized in a single question: "How many days in the last 3 months were you at least $50 \%$ disabled due to migraine at work, home, school, or recreational activities?" Each patient can be assigned to one of four disability levels, from grade I (corresponding to little or no disability) to grade IV (corresponding to severe disability), on the basis of standardized intervals of the MIDAS total score which is obtained by the simple sum of the scores of the five questions.

HIT6 includes 6 questions in different domains concerning severity of pain, limitations in activities, tiredness, and mood changes related to migraine in the past four weeks. Each answer requires a rating on a 5-point scale (from never to always). Total score ranges from 36 to 78, with higher scores indicating a greater headache impact on patients. A score between 36 and 49 indicates little or no impact, a score of 60 or more indicates very severe headache-related impact.

Thus, both MIDAS and HIT6 may be very useful tools to verify the clinical situation from the patient point of view, providing a measurable insight on the global effect of prophylaxis by simple paper-and-pencil questionnaires.

Efficacy may be the most relevant factor guiding patient preferences in prevention. In a recent study (Peres et al 2007), $72 \%$ of enrolled patients rated effectiveness as the most relevant positive aspect of prophylaxis, chosen from several factors. Furthermore, when patients were invited to choose among several different hypothetical prophylaxis scenarios (each one containing a simulation of 2 hypothetical headache 
preventive treatments with different characteristics), most of them were likely to select those preventive treatments with higher efficacy rates, possibly with fewer adverse events, but still preferring treatments with higher efficacy rates even if side effects were present. In this study (Peres et al 2007), however, "efficacy" might refer only to frequency reduction, and other aspects (such as changes in functioning and in quality of life, or in severity of pain or acute therapy efficacy) were not explored.

\section{Possibility of adverse events}

The occurrence of adverse events following administration of preventive drugs is a very common concern expressed by migraine patients when they receive the prescription of prophylaxis. Concern for side effects may discourage sufferers from accepting prophylaxis or may negatively influence patient adherence, causing different problems, such as non regular daily dosing, self-reduction of the prescribed compound, or withdrawal of prophylaxis following trivial symptoms or events which are not treatment-related.

To overcome these problems, a comprehensive history taking should include: evaluation of life style (occupation, sports, time schedules, etc.) of the individual patient, eliciting fear of a particular adverse event, and obtaining history of occurrence of side effects from previous treatments. Clinicians should choose the preventive compound accordingly, with the active participation of their patient.

In any case, patients should be advised about the most common adverse events of the prescribed drugs and even of those uncommon effects which could be clinically relevant and which might require drug withdrawal. If possible, focus should be on the benign nature of most adverse events, the possibility that they could be reduced during the course of treatment, or that they could be minimized by reducing the daily doses.

The following scenarios can be regarded as practical examples. Compounds with a high risk of increasing weight (such as flunarizine, valproate, or amitriptyline) should be avoided in a patient who is very concerned with weight gain. A drug which can cause difficulty in memory or speech slowing (such as topiramate) should be not the first choice in a patient who works as a teacher, or the possible occurrence of these effects should be communicated, instructing the patient to taper down the daily dose and eventually to stop treatment if these effects occur. Drugs which are likely to cause excessive sedation or somnolence (such as amitriptyline) should not be prescribed to a taxi driver.

We suggest informing patients that symptoms such as paresthesias during topiramate treatment, mild somnolence with flunarizine, or some nausea with sodium valproate should be regarded as substantially benign and often self-limiting or gradually vanishing adverse events: On the other hand, patients should be told to contact their physician if they experience visual disturbances, depression, or tremor while on topiramate, flunarizine, or sodium valproate, respectively.

The recent survey among 250 patients from two headache centers in the US and in Brazil with primary headache diagnoses (Peres et al 2007) suggests that fear of adverse events may be less relevant in patient preference and eventually in acceptance. Only $6 \%$ of the enrolled headache patients rated absence of adverse events as the most important aspect of prevention when they were asked to rank a list of aspects which also included efficacy, speed of onset, frequency of dosing, out-of-pocket expenses, and type of treatment (prescription/ vitamin). In this study (Peres et al 2007), however, only a few side effects were suggested for the patient evaluation, that is, weight gain, concentration difficulty, and fatigue.

In another study on a clinical sample (150 individuals with a history of migraine at a US headache center) (Rozen 2006), migraine patients were asked to rank on a 1 to 10 scale some characteristics of preventive treatments. The second most highly ranked factor in terms of importance was that the prescribing clinician take time to explain the possible adverse events of the proposed medications. Again, data obtained in this sample suggest that migraine patients would accept a preventive treatment with a high side effect rate if it is effective. In the same study, possible effects on weight and the likelihood to cause sedation were indicated as important factors as to why patients (especially women) might not accept migraine prophylaxis.

\section{Concluding remarks}

The management of migraine is not a simple task, and clinicians should be aware that treatment strategies for migraine patients require that they understand and accept treatment decisions. Education is important for any therapeutic intervention in migraine (such as acute therapy, avoidance of trigger factors, and non-pharmacological approaches), but may be particularly relevant when a long-term treatment plan, including preventive therapy, is prescribed.

In fact, prophylaxis requires a comprehensive evaluation of different aspects of a patient's migraine and life style such as frequency and severity of attacks, response to acute therapy, quality of life and migraine-related functional disability, and life style and specific needs, to guide therapeutic decision, as well as to assess the outcome of the selected treatment. In this context, a patient's acceptance of preventive 
therapy could be negatively influenced by several factors. Among these, reluctance to take medications every day and for long periods, concern about possible adverse events, and unrealistic expectations are the most common.

Clinicians must direct their efforts to help their patients accept prophylaxis, taking into account the following suggestions:

- Differences in goals and treatment schedules between acute therapy (meant to address the immediate need for relief during an attack), and prophylaxis (whose main goals are to reduce the frequency of headache episodes as well as to limit the negative effects of migraine on daily life) should be clearly explained, in order to help patients understand that preventive drugs must be taken every day, and for long periods.

- Accurate evaluation of the global clinical situation and life style of the individual patient should be performed.

- The fact that the information given by the patient (particularly on those adverse events that could be intolerable and on the presence of concomitant conditions which might influence treatment course) has been taken into account to select the most appropriate prophylaxis must be communicated to the patient.

- Active involvement of the individual patient in the assessment of prophylaxis outcomes during the treatment period should be reached, through the regular use of a headache diary and through the administration of specific disability/impact tools, with a consequent likely increase in the patient's adherence with treatment schedule and satisfaction.

We agree with other authors (Rozen 2006; Manzoni and Torelli 2007) about the crucial role of the communication between patient and physician in the therapeutic management of primary headaches. This is particularly true when preventive therapy is indicated, when the patient-physician interaction may count more than the characteristics of a single preventive compound.

\section{Future research}

While patient preference for acute migraine treatment has been extensively studied (Salonen et al 1999; Powers et al 2000; Davies et al 2000; Sheftell et al 2004; Dowson et al 2004; Goadsby et al 2004; Láinez et al 2006; Díez et al 2007), only three studies have been published on patient preference for prophylaxis (Rozen 2006; Peres et al 2007; Kol et al 2008). Their results give an insight on patient attitudes and expectations in migraine prevention, but further studies are warranted to explore all the possible factors influencing patient acceptance, possibly in clinical samples and in population samples. Pharmacological research should be aimed at developing new preventive compounds with fewer adverse events in order to favor patient acceptance.

Another challenge for the near future is creating a more widespread awareness and knowledge about the importance of migraine as a public health problem. It has been well established that most people with migraine do not receive a correct diagnosis and are likely to be inadequately treated (Lipton et al 2001; Lipton et al 2003; MacGregor et al 2003; Lucas et al 2005; Lucas et al 2006; Diamond 2006; Lipton et al 2007). In surveys on the general population performed in France (Lucas et al 2006), in the US (Lipton et al 2007), and in the Netherlands (Terwindt et al 2000), the proportion of migraineurs not aware of their diagnosis ranged from $47 \%$ to $70 \%$. Understandably, a corresponding underutilization of specific anti-migraine compounds, and particularly preventive drugs, is evident in different countries. In fact, based on indications provided by international guidelines, up to $38 \%$ to $50 \%$ of patients with migraine may be considered candidates for prevention (D'Amico and Lanteri-Minet 2006; Lipton et al 2007), but only a minority of them are on prophylaxis ( $6 \%$ to $13 \%$ in population-based surveys) (Lucas et al 2005; Lipton et al 2007). Thus, a substantial proportion of migraine sufferers who might benefit from prevention do not receive it, and it is likely that many of them would reject treatment due to the problems with acceptance of prophylaxis described above, further reducing the numbers of migraineurs appropriately treated.

More importantly, the authors believe that educational programs directed to health care professionals (such as primary care physicians, neurologists, nurse practitioners, and physician assistants) must be encouraged. Clinicians should be made aware that migraine is a clinically significant disorder that, although generally benign, may cause disruption of individual patient lives, and that underdiagnosis and inappropriate treatment may cause increase in attack frequency, with consequent overuse of acute drugs, poor patient outcome and satisfaction. These factors are likely to increase the risk of migraine chronification and of the development of medication overuse headache and chronic daily headache (Mathew et al 1982; Silberstein et al 1996; Scher et al 2003; Katsarava et al 2004). Chronic migraine, chronic daily headache, and medication overuse headache will, in turn, cause a more disabling burden on patient lives, and are more difficult to treat (Monzón et al 1998; Meletiche et al 2001; Guitera et al 2002; Bigal et al 2003; D'Amico et al 2003; D'Amico et al 2004; D'Amico et al 2005; D'Amico et al 2008). 
Education should also be focused on the availability of the options for effective prophylaxis, as well as on emphasizing that good communication between physician and patient is the key factor in successful long-term therapy. Through this communication, patients can express their needs and become actively involved in the decision-making process of choosing whether to use migraine prophylaxis and how to select the most appropriate preventive compound. The active participation of patients improves acceptance of physician prescriptions. Of course, the role of the treating clinician is essential in each step of migraine management, putting into practice knowledge of published evidence and clinical experience, providing correct information and education, and setting realistic expectations for possible adverse events and for treatment goals in a given migraine patient.

\section{Disclosures}

Dr D'Amico - in the last 5 years, grants and honoraria for participating in meetings/congresses or Scientific Boards from AstraZeneca, Merck, Janssen-Cilag, GSK, OrthoMcNeil, Pfizer.

Dr Tepper: grants/research support from Allergan, Alexza, ANS/Advanced Bionics, AstraZeneca, Eisai, Endo, Forrest, GSK, King, Merck, Medtronix, Minster, Neurochem, NMT, Novartis, Nupathe, OrthoMcNeil, Pfizer, Pozen, Proethic, Takeda, Winston, Vernalis.

Consultant in last 5 years (only those marked 2007-2008 current): Allergan (2007), AstraZeneca, Coherex (2008), Elan, Endo (2007-8), Forrest, GSK (2007-8), Merck (2007-8), NMT (2007-8), OrthoMcNeil (2007), Vernalis.

Speakers Bureau (only those marked 2007-2008 current): Allergan, AstraZeneca, Endo (2007), GSK (2008), Merck (2008), NMT (2008), OrthoMcNeil (2007), Pfizer, Valeant (2008).

Advisors Board, 2007-8 GSK, Merck.

\section{References}

Ayata C, Jin H, Kudo C, et al. 2006. Suppression of cortical spreading depression in migraine prophylaxis. Ann Neurol, 59:652-61.

Bigal ME, Rapoport AM, Lipton RB, et al. 2003. Assessment of migraine disability using the migraine disability assessment (MIDAS) questionnaire: a comparison of chronic migraine with episodic migraine. Headache, 43:336-42.

Bigal ME, Lipton RB. 2006. Modifiable risk factors for migraine progression (or for chronic daily headaches) - clinical lessons. Headache, 46 (Suppl 3):S144-6.

Blau JN. 1980. Migraine prodromes separated from the aura: Complete migraine. BMJ, 281:658-60.

Blau JN. 1984. Resolution of migraine attacks: Sleep and the recovery phase. J Neurol Neurosurg Psychiatry, 47:437-42.

Brandes JL, Saper JR, Diamond M, et al; for the MIGR-002 Study Group. 2004. Topiramate for migraine prevention: a randomized controlled trial. JAMA, 291(8):965-73.
Brandes JL, Kudrow DB, Rothrock JF, et al. 2006. Assessing the ability of topiramate to improve the daily activities of patients with migraine. Mayo Clin Proc, 81(10):1311-9.

Dahlof CGH, Dimenas E. 1995. Migraine patients experience poorer subjective well-being/quality of life even between attacks. Cephalalgia, 15:31-6.

Dahlof C, Loder E, Diamond M, et al. 2007. The impact of migraine prevention on daily activities: a longitudinal and responder analysis from three topiramate placebo-controlled clinical trials. Health Qual Life Outcomes, 4;5(1):56.

D'Amico D, Usai S, Grazzi L, et al. 2003. Quality of life and disability in primary chronic daily headaches. Neurol Sci, 24:S97-S100.

D'Amico D, Usai S, Grazzi L, et al. 2004. The impact of primary headaches on patients' lives: Italian experience with the MIDAS and the SF-36 questionnaires. Headache Care, 1:123-8.

D’Amico D, Grazzi L, Usai S, et al. 2005. Disability pattern in chronic migraine with medication overuse: a comparison with migraine without aura. Headache, 45:553-60.

D'Amico D, Lanteri-Minet M. 2006. Migraine preventive therapy: selection of appropriate patients and general principles of management. Expert Rev Neurother, 6:1147-57.

D'Amico D, Leone M, Grazzi L, et al. 2008. When should "chronic migraine" patients be considered "refractory" to pharmacological prophylaxis? Neurol Sci, 29(Suppl 1):S55-58.

Davies GM, Santanello N, Lipton RB. 2000. Determinants of patient satisfaction with migraine therapy. Cephalalgia, 20:554-60.

Diamond ML, Wenzel RG, Nissan GR. 2006. Optimizing migraine therapy: evidence-based and patient-centered care. Expert Rev Neurother, 6:911-9.

Diez FI, Straube A, Zanchin G. 2007. Patient preference in migraine therapy. A randomized, open-label, crossover clinical trial of acute treatment of migraine with oral almotriptan and rizatriptan. J Neurol, 254:242-9.

Dowson AJ, Sender J, Lipscombe S, et al. 2003. Establishing principles for migraine management in primary care. Int J Clin Pract, 57:493-507.

Dowson AJ, Tepper SJ, Baos V, et al. 2004. Identifying patients who require a change in their current acute migraine treatment: the Migraine Assessment of Current Therapy (Migraine-ACT) questionnaire. Current Med Res Opin, 20:1125-35.

Dowson AJ, Tepper SJ, Dahlof C. 2005. Patients' preference for triptans and other medications as a tool for assessing the efficacy of acute treatments for migraine. $J$ Headache Pain, 6:112-20.

Diamond S, Bigal ME, Silberstein S, et al. 2007. Patterns of diagnosis and acute and preventive treatment for migraine in the United States: results from the American Migraine Prevalence and Prevention Study. Headache, 47:355-63.

D'Amico D, Solari A, Usai S, et al; for the Progetto Cefalee Lombardia Group. 2006. Improvement in quality of life and activity limitations in migraine patients after prophylaxis. A prospective longitudinal multicentre study. Cephalalgia, 26:691-96.

Diener HC, Tfelt Hansen P, Dahlof C, et al; for the MIGR-003 Study Group. 2004. Topiramate in migraine prophylaxis - results from a placebo-controlled trial with propranolol as an active control. J Neuro, 251:943-50.

Diener HC, Agosti R, Allais G, et al; for the TOPMAT-MIG-303 Investigators Group. 2007. Cessation versus continuation of 6-month migraine preventive therapy with topiramate (PROMPT): a randomised, doubleblind, placebo-controlled trial. Lancet Neurol, 6:1054-62.

Dueland AN, Leira R, Burke TA, et al. 2004. The impact of migraine on work, family, and leisure among young women - a multinational study. Curr Med Res Opin, 20:1595-604.

Goadsby PJ, Dodick DW, Ferrari MD, et al. 2004. TRIPSTAR: prioritizing oral triptan treatment attributes in migraine management. Acta Neuro. Scand, 110:137-43.

Guitera V, Munoz P, Castillo J, et al. 2002. Quality of life in chronic daily headache: a study in a general population. Neurology, 58:1062-5.

Gursoy-Ozdemir Y, Qiu J, Matsuoka N, Bolay H, et al. 2004. Cortical spreading depression activates and upregulates MMP-9. J Clin Invest, 113:1447-55. 
Headache Classification Subcommittee of the International Headache Society. 2004. The international classification of headache disorders: 2nd edition. Cephalalgia, 24(Suppl 1):1-160.

Henry P, Auray JP, Gaudin AF et al. 2002. Prevalence and clinical characteristics of migraine in France. Neurology, 59:232-7.

Joffe H, Cohen LS, Suppes T, et al. 2006. Valproate is associated with new-onset oligoamenorrhea with hyperandrogenism in women with bipolar disorder. Biol Psychiatry, 59:1078-86.

Katsarava Z, Schneeweiss S, Kurth T, et al. 2004. Incidence and predictors for chronicity of headache in patients with episodic migraine Neurology, 62:788-90.

Klapper J. 1997. Divalproex sodium in migraine prophylaxis: a dosecontrolled study. Cephalalgia, 17:103-38.

Kol CM, Dekker F, Neven AK, et al. 2008. Acceptance or rejection of prophylactic medicine in patients with migraine: a cross-sectional study. Br J Gen Pract, 58:98-101.

Kosinski M, Bayliss MS, Bjorner JB, et al. 2003. A six-item short-form survey for measuring headache impact: the HIT-6. Qual Life Res, 12:963-74.

Lainez MJ, Evers S, Kinge E, et al. 2006. Preference for rizatriptan 10-mg wafer vs. eletriptan $40-\mathrm{mg}$ tablet for acute treatment of migraine. Cephalalgia, 26:246-56.

Launer LJ, Terwindt GM, Ferrari MD. 1999. The prevalence and characteristics of migraine in a population-based cohort. Neurology, 53:537-42.

Lipton RB, Stewart WF, Diamond S, et al. 2001. Prevalence and burden of migraine in the United States: data from the American Migraine Study II. Headache, 41:646-57.

Lipton RB, Bigal ME, Kolodner K, et al. 2003. The family impact of migraine: population-based studies in the USA and UK. Cephalalgia, 23:429-40.

Lipton RB, Sher AI, Steiner TJ, et al. 2003. Patterns of health care utilization for migraine in England and in the United States. Neurology, 60:441-8.

Lipton RB, Göbel H, Einhäupl KM, et al. 2004. Petasites hybridus root (butterbur) is an effective preventive treatment for migraine. Neurology, 63:2240-4.

Lipton RB, Bigal ME, Diamond M, et al. 2007. AMPP Advisory Group. Migraine prevalence, disease burden, and the need for preventive therapy. Neurology, 68:343-9.

Lucas C, Chaffaut C, Artaz MA, et al. 2005. FRAMIG 2000: medical and therapeutic management of migraine in France. Cephalalgia, 25:267-79.

Lucas C, Géraud G, Valade D, et al. 2006. Recognition and therapeutic management of migraine in 2004, in France: results of FRAMIG 3, a French nationwide population-based survey. Headache, 46:715-25.

MacGregor E A, Brandes J, Eikermann A. 2003. Migraine Prevalence and Treatment Patterns: The Global Migraine and Zolmitriptan Evaluation Survey. Headache, 43:19-26.

Manzoni CG, Torelli P. 2007. The patient-physician relationship in the approach to therapeutic management. Neurol Sci, 28:S130-S133.

Mathew NT, Stubits E, Nigam MR. 1982. Transformation of migraine into daily headache: analysis of factors. Headache, 22:66-8.

Mathew NT, Rapoport A, Saper J, et al. 2001. Efficacy of gabapentin in migraine prophylaxis. Headache, 41:119-28.

Meletiche DM, Lofland JH, Young WB. 2001. Quality-of life differences between patients with episodic and transformed migraine. Headache, 41:573-8.

Members of the task force: Evers S, Afra J, Frese A, et al. 2006. EFNS guideline on the drug treatment of migraine - report of an EFNS task force. Eur J Neurol, 13:560-72.

Monzón MJ, Láinez MJ. 1998. Quality of life in migraine and chronic daily headache patients. Cephalalgia, 18:638-43.

Osterhaus JT, Townsend RJ, Gandek B, et al. 1994. Measuring the functional status and well-being of patients with migraine headache. Headache, 34:337-43.

Peres MF, Silberstein S, Moreira F, et al. 2007. Patients' preference for migraine preventive therapy. Headache, 47:540-5.

Powers C, Szeto S, Pangtay D, et al. 2000. Evaluation of migraineurs' preferences for naratriptan over conventional first-line agents. Arch Fam Med, 9:753-8.
Ramadan NM, Silberstein SD, Freitag FG, et al. 2000. Evidence-based guidelines for migraine headache in the primary care setting: pharmacological management for prevention of migraine. Neurology, (serial on line) www.neurology.org.

Rapoport A, Mauskop A, Diener HC, et al. 2006. Long-term migraine prevention with topiramate: open-label extension of pivotal trials. Headache, 46:1151-60.

Roncolato M, Fabbri L, Recchia G, et al. 2000. An epidemiological study to assess migraine prevalence in a sample of Italian population presenting to their GPs. Eur Neurol, 43:102-6.

Roth BL. 2007. Drugs and valvular heart disease. N Engl J Med, 356:6-9.

Rozen TD. 2006. Migraine prevention: what patients want from medication and their physicians (a headache specialty clinic perspective). Headache, 46:750-3.

Salonen R, Ashford EA, Gibbs M, et al. 1999. Patient preference for oral sumatriptan $25 \mathrm{mg}, 50 \mathrm{mg}$, or $100 \mathrm{mg}$ in the acute treatment of migraine: a double-blind, randomized, crossover study. Sumatriptan Tablets S2CM11 Study Group. Int J Clin Pract, (Suppl)105:16-22.

Sándor PS, Afra J, Ambrosini A, et al. 2000. Prophylactic treatment of migraine with beta-blockers and riboflavin: differential effects on the intensity dependence of auditory evoked cortical potentials. Headache, 40:30-5.

Scher AI, Stewart WF, Ricci JA, et al. 2003. Factors associated with the onset and remission of chronic daily headache in a population-based study. Pain, 106:81-9.

Sheftell FD, Feleppa M, Tepper SJ, et al. 2004. Patterns of use of triptans and reasons for switching them in a tertiary care migraine population. Headache, 44:661-8.

Silberstein SD, Silberstein MM. 1990. New concepts in the pathognesis of headache-Part II. Pain Manag, 3:334-42.

Silberstein SD, Lipton RB, Sliwinski M. 1996. Classification of daily and near-daily headaches: field trial of revised IHS criteria. Neurology, 47:871-5.

Silberstein SD; for the US Headache Consortium. 2000. Practice parameter: Evidence-based guidelines for migraine headache (an evidence-based review). Report of the Quality Standars Subcommittee of the American Academy of Neurology. Neurology, 55:754-62.

Silberstein SD, Goadsby PJ. 2002. Migraine: preventive treatment Cephalalgia, 22:491-512.

Silberstein SD, Neto W, Schmitt J, et al; and the MIGR-001 Study Group. 2004. Topiramate in migraine prevention: results of a large controlled trial. Arch Neurol, 61:490-5.

Silberstein SD, Dodick D, Freitag F, et al. 2007. Pharmacological approaches to managing migraine and associated comorbidities - clinical considerations for monotherapy versus polytherapy. Headache, 47:585-99.

Steiner TJ, Scher AI, Stewart WF, et al. 2003. The prevalence and disability burden of adult migraine in England and their relationships to age, gender and ethnicity. Cephalalgia, 23:519-27.

Stewart WF, Sheder AL, Simon D, et al. 1994. Migraine prevalence. A review of population-based studies. Neurology, 44(Suppl 4):S17-S23.

Stewart WF, Shechter A, Lipton RB. 1994. Migraine heterogeneity. Disability, pain intensity, and attack frequency and duration. Neurology, 44(6 Suppl 4):S24-33

Stewart WF, Lipton RB, Whyte J, et al. 1999. An international study to assess reliability of the Migraine Disability Assessment (MIDAS) score. Neurology, 53:988-94

Tepper SJ, D'Amico D, Baos V, et al. 2004. Guidelines for prescribing prophylactic medications for migraine: a survey among headache specialists in different countries. Headache Care, 1:267-72.

Tepper SJ, Bigal, M, Rapoport A, Sheftell F. Alternative therapies: evidence based evaluation in migraine. Headache Care, 2006, 3:57-64.

Terwindt GM, Ferrari MD, Tijhuis M, et al. 2000. The impact of migraine on quality of life in the general population. Neurology, 55:624-9.

Wang SJ. 2003. Epidemiology of migraine and other types of headache in Asia. Curr Neurol Neurosci Rep, 3:104-8.

Ziad el K, Rahi AC, Hamdan SA, et al. 2005. Age, dose, and environmental temperature are risk factors for topiramate-related hyperthermia. Neurology, 65:1139-40. 
